\title{
Review Article \\ Role of Farnesoid X Receptor in the Pathogenesis of Respiratory Diseases
}

\author{
Jin-nan Wu $\mathbb{D}^{1},{ }^{1}$ Jian-rong Chen $\mathbb{D}^{\circ},{ }^{2}$ and Jin-liang Chen $\mathbb{D D}^{2}$ \\ ${ }^{1}$ Postgraduate in Respiratory Medicine, Affiliated Hospital 2 of Nantong University, Nantong, China \\ ${ }^{2}$ Department of Respiratory Medicine, Affiliated Hospital 2 of Nantong University, Nantong, China \\ Correspondence should be addressed to Jian-rong Chen; drchenjr@163.com and Jin-liang Chen; cjllcx123@126.com
}

Received 27 June 2020; Revised 2 November 2020; Accepted 13 November 2020; Published 26 November 2020

Academic Editor: Andrea S. Melani

Copyright (c) 2020 Jin-nan Wu et al. This is an open access article distributed under the Creative Commons Attribution License, which permits unrestricted use, distribution, and reproduction in any medium, provided the original work is properly cited.

Farnesoid X receptor (FXR) is a bile acid receptor encoded by the Nr1h4 gene. FXR plays an important role in maintaining the stability of the internal environment and the integrity of many organs, including the liver and intestines. The expression of FXR in nondigestible tissues other than in the liver and small intestine is known as the expression of "nonclassical" bile acid target organs, such as blood vessels and lungs. In recent years, several studies have shown that FXR is widely involved in the pathogenesis of various respiratory diseases, such as chronic obstructive pulmonary disease, bronchial asthma, and idiopathic pulmonary fibrosis. Moreover, a number of works have confirmed that FXR can regulate the bile acid metabolism in the body and exert its antiinflammatory and antifibrotic effects in the airways and lungs. In addition, FXR may be used as a potential therapeutic target for some respiratory diseases. For example, FXR can regulate the tumor microenvironment by regulating the balance of inflammatory and immune responses in the body to promote the occurrence and development of non-small-cell lung cancer (NSCLC), thereby being considered a potential target for immunotherapy of NSCLC. In this article, we provide an overview of the internal relationship between FXR and respiratory diseases to track the progress that has been achieved thus far in this direction and suggest potential therapeutic prospects of FXR in respiratory diseases.

\section{Introduction}

Farnesoid X receptor (FXR) is a ligand-activated transcription factor encoded by the Nrlh4 gene; this transcription factor belongs to the bile acid receptor of the nuclear receptor superfamily [1]. FXR plays a vital role in maintaining the stability of the internal environment and the integrity of various organs, including the liver and intestines. A large number of studies have demonstrated that FXR plays an important role in the metabolism of bile acid [2], cholesterol [3], lipids [4], and glucose [5] and the regulation of intestinal flora [6]. It is also widely involved in the occurrence and development of cholestasis [7], alcoholic liver disease [8], primary biliary cirrhosis [9], inflammatory bowel disease [10], atherosclerosis [11], and even gastrointestinal tract and other malignant tumors [12]. In addition, FXR can be used as a potential therapeutic target for several diseases $[13,14]$. Recent studies have suggested that FXR may play a considerable role in the physiological and pathological aspects of the respiratory system [15-17]. In this article, we provide an overview of the internal relationship between FXR and respiratory diseases to track the progress that has been achieved thus far in this direction and suggest potential therapeutic prospects of FXR in respiratory diseases.

\section{Biological Characteristics of FXR}

2.1. Distribution and Structure of FXR. FXR was isolated by Forman for the first time in a rat liver cDNA library [18]. FXR was initially considered as a simple orphan nuclear receptor. However, bile acid metabolites were observed to have the ability to fully bind to FXR at the physiological level. Hence, FXR was finally identified as a bile acid receptor. FXR is presently considered as a kind of bioreceptor for bile acid receptors and synthesis that fully participates in the regulation of bile acid metabolism. FXR plays an important role 
in maintaining the stability of the internal environment and the integrity of various organs, including the liver and intestines $[19,20]$.

FXR has two known genotypes, namely, FXR $\alpha$ (NR1H4) and FXR $\beta$ (NR1H5). Since its discovery, the FXR gene has been successfully cloned in numerous species, including humans, rats, and mice. The single $\operatorname{FXR} \alpha$ gene in humans and rodents encodes four different isoforms, namely, $\mathrm{FXR} \alpha 1, \mathrm{FXR} \alpha 2, \mathrm{FXR} \alpha 3$, and $\mathrm{FXR} \alpha 4$, which are caused by different promoters and RNA splicing, and their expression is tissue specific [21]. However, $\operatorname{FXR} \beta$ is expressed as a pseudogene in human and primates, and its mechanism of action remains unclear $[22,23]$. Therefore, FXR is used to express FXR $\alpha$ in this review. The human FXR gene is located on chromosome 12 (12q23.1), which is mainly expressed in various digestible tissues, including the liver [24] and small intestine [25]. In addition, the expression of FXR in nondigestible tissues other than in the liver and small intestine is known as the expression of "nonclassical" bile acid target organs, such as kidneys [26], adrenal glands [27], lung tissue [13], and blood vessels [28].

As an orphan nuclear receptor, FXR has a typical nuclear receptor structure [29]. Its structure includes highly conserved carboxy-terminal ligand-binding domain (LBD), amino-terminal DNA-binding domain (DBD), low conserved amino-terminal ligand-independent transcription activation functional domain (AF-1), carboxy-terminal ligand-dependent transcription activation functional area (AF-2), and hinge area connecting LBD and DBD. FXR is a ligand-activated transcription factor classified as a nuclear bile acid receptor. Under the action of a ligand, the FXR that is activated by an agonist (such as bile acid) enters the nucleus of the cells and then combines with retinoid $X$ receptor to form a heterodimer, which can be bound to a specific FXR response elements and regulate the expression of various target genes by changing the structural conformation of FXR [30, 31].

2.2. Function of FXR. FXR was first widely recognized for regulating the bile acid pathway in the liver and intestines. Previous studies have demonstrated that FXR regulates the bile acid synthesis through two main pathways [32-34]. In the classical pathway, CYP7A1 is a liver-specific microsomal cytochrome $\mathrm{P} 450$ enzyme, which is a rate-limiting enzyme in the classic pathway of bile acid synthesis. CYP7A1 is only expressed in the human liver and can catalyze the $7 \alpha$ hydroxylation of cholesterol. In the alternative pathway, CYP27A1 initiates an alternative pathway of bile acid synthesis, in which cholesterol is oxidized to form chenodeoxycholic acid. The main physiological function of FXR is to regulate the conversion of cholesterol into bile acid, which is the basis for the body to participate in other biochemical reactions.

Recent studies have reported that FXR plays an important role in regulating lipid and glucose metabolism $[4,5]$. Moreover, FXR plays a vital role in preventing disorder infections of intestinal flora and formation of biliary calculus $[6,7]$. FXR activation exhibits beneficial effects on various metabolic diseases, including fatty liver [35], type 2 diabetes [36], and hyperlipidemia [37]. Accumulating evidence supports the observation that FXR agonists are beneficial to the regulation of enterohepatic circulation [38], liver regeneration [39], and occurrence and development of metabolic tumors, such as liver cancer [40]. Moreover, FXR agonists prevent the development of atherosclerosis [11] and kidney diseases [41], and they are valuable for the treatment of diabetes, hyperlipidemia, and obesity [42, 43]. These properties indicate that FXR activation has a systemic effect and may be a potential strategy for the treatment of a variety of disorders.

\section{FXR and Respiratory Diseases}

Increasing evidence shows that the expression of FXR in "nonclassical" bile acid target tissues, such as blood vessels and lungs $[15,28]$, is equivalently important. In the vasculature, FXR regulates cholesterol transport and vascular tension by regulating its own expression [44, 45]. Hendrick et al. confirmed the presence of FXR in human airway epithelial cell lines and pulmonary vascular endothelial cell lines [46]. Zhang et al. [47] established that FXR could inhibit lipopolysaccharide- (LPS-) induced acute lung inflammation injury, as well as regulate and improve lung regeneration in animal experimental models. Several studies have reported that FXR agonists, such as 6-ethylchenodeoxycholic acid (OCA), play an anti-inflammatory and antifibrotic role in lung tissues, and FXR can be used as a new target for the treatment of certain lung diseases $[13,48]$. This review provides an overview of these studies and then summarizes the latest progress in FXR research on respiratory diseases.

3.1. Chronic Obstructive Pulmonary Disease. Chronic obstructive pulmonary disease (COPD) is a common and frequently occurring respiratory disease, with high morbidity and mortality. COPD is characterized by continuous and mostly progressive airflow limitation mainly due to abnormal airway remodeling and chronic inflammation. Lesions in small airways, such as small airway inflammation, fibrous tissue formation, and lumen mucus plugs, can lead to increased peripheral airway resistance in patients with COPD [49]. Although the mechanism of airway remodeling in COPD has not been fully clarified, the process involves epithelial-mesenchymal transition (EMT) [50], the development of which is related to the severity of airway restriction [51]. In addition, cyclooxygenase 2 (COX-2) is a key enzyme involved in converting arachidonic acid to prostaglandin E2, which is an important factor leading to the airway inflammation of COPD. In this manner, the expression of COX-2 is also related to the severity of airflow limitation $[52,53]$.

Chen et al. [54] detected FXR expression in human small airway epithelial cells and also in the rat COPD lung model. They found that FXR could promote bile acid-induced EMT in alveolar epithelial cell lines, suggesting that the overexpression of FXR may lead to airflow limitation in patients 
with COPD by promoting EMT in the small airways. Furthermore, they observed that COX-2 is overexpressed in the small pulmonary airways of COPD, and bile acid inhalation may promote airway inflammation through FXR-mediated COX-2, which in turn results in continuous airflow limitation of COPD. However, FXR could also inhibit LPS-induced acute lung inflammation [47]. This contradictory effect of FXR may be related to various causes of respiratory diseases, a research direction that requires further investigation. Nevertheless, FXR does participate in the pathogenesis of COPD.

3.2. Asthma. Asthma is among the most common chronic diseases worldwide. It is a chronic inflammatory disease of the airways involving multiple types of cells and cell components [55]. It is characterized by airway inflammation, hyperresponsiveness, and remodeling. On the one hand, chronic airway inflammation of asthma is due to the activated helper Th2 cells that produce interleukins, such as interleukin-4 (IL-4), interleukin-5 (IL-5), and interleukin-13 (IL-13), to activate B lymphocytes, synthesize specific immunoglobulin $\mathrm{E}$, and activate mast cells and eosinophils to mediate the synthesis and release of a variety of active mediators [56]. On the other hand, the production of cytokines, such as interleukins, by activated helper Th2 cells can directly activate mast cells, eosinophils, and alveolar macrophages, which in turn can secrete a variety of inflammatory mediators and cytokines, such as histamine, leukotrienes, prostaglandins, and transforming growth factors $[57,58]$. In addition, nuclear factor-kappaB (NF- $\kappa \mathrm{B})$, as an important nuclear transcription factor in the cell, is widely involved in the inflammatory and immune responses, can regulate cell apoptosis and stress response, and plays an important role in the pathogenesis of asthma [59, 60].

Shaik et al. [61] found that FXR mRNA and protein can be expressed in mouse lung tissues and in rat and human lung endothelial cells, indicating that FXR is involved in normal physiological responses in lung tissues. In the constructed ovalbumin-induced acute rat asthma model, they found that the anti-inflammatory effect of FXR is achieved by blocking the infiltration of inflammatory cells into normal lung tissues, thereby inhibiting the secretion of IL-4, IL- 5 , and IL- 13 by activated helper Th 2 cells and that of tumor necrosis factor- $\alpha$ (TNF- $\alpha$ ) by activated alveolar macrophages. Moreover, they noted that FXR may partially reduce airway inflammation in asthma by antagonizing NF$\kappa \mathrm{B}$ signaling and target gene expression in vivo.

3.3. Idiopathic Pulmonary Fibrosis. Idiopathic pulmonary fibrosis (IPF) is a chronic, progressive, and fibrotic interstitial pneumonia [62]. It has typical histopathological features or radiographic images of common interstitial pneumonia. The pathogenesis of IPF is not completely clear. IPF may be the result of the interaction between genetic and environmental factors. From the perspective of pathophysiology, IPF is presently understood to originate from abnormal repair of alveolar epithelium after repeated minor injury [63]. Repeated minor injury leads to the apoptosis of alveolar epithelium, and abnormal activation of epithelium induces the proliferation of intrinsic fibroblasts which stimulate the development of EMT and the differentiation of fibroblasts into myofibroblasts. These processes in turn lead to progressive pulmonary interstitial fibrosis and airway stiffness that can cause breathing difficulties and eventually result in respiratory failure. EMT, inflammatory processes, and collagen deposition are considered as important mechanisms in the pathogenesis of IPF [64, 65].

EMT is a process, in which fully differentiated epithelial cells gradually transform into mesenchymal phenotypes that involves the activation of transforming growth factor- $\beta 1$ (TGF- $\beta 1$ ), connective tissue growth factor, epidermal growth factor, and platelet-derived growth factor subunit A [66]. The common feature of these pathways is that they have the ability to promote the activation of "major transcription factors," such as zinc finger transcription factors 1 (SNAI1) and zinc finger transcription factors 2 (SNAI2), which are responsible for initiating the EMT program in epithelial cells $[67,68]$.

FXR is not only located in alveolar epithelial type I cells (AECIs) but also in alveolar epithelial type II cells (ATECIIs) [69]. ATII cells are multifunctional cells that synthesize and secrete lung surfactants and can participate in the immune response by producing AECIs [70]. In addition, the expression of FXR in ATECIIs is regarded as the main source of mesenchymal expansion in pulmonary fibrosis [71]. Comeglio et al. $[13,72]$ reported that TGF- $\beta 1$, SNAI1, and SNAI2 expression levels substantially increase in the constructed rat model of pulmonary fibrosis, whereas, with the participation of FXR agonists (such as OCA), their expression levels are restored to those of the control group, thereby inhibiting the development of EMT. Several studies have proposed that IL- 6 is a key factor that mediates the proliferation of fibroblasts driven by TGF- $\beta 1$ in the lungs likely by converting acute inflammation of the lungs to a more chronic fibrotic state [73]. Comeglio et al. confirmed that OCA could considerably reduce the production of proinflammatory cytokines (i.e., IL- $1 \beta$, IL- 6 , and TNF- $\alpha$ ) in the IPF rat model and could also regulate the proportion of matrix metalloproteinases (MMP) and their inhibitors (tissue inhibitor of metalloproteinases, TIMP), thereby alleviating the symptoms of pulmonary fibrosis. Therefore, FXR inhibits the occurrence of IPF mainly via two avenues: it inhibits the production of proinflammatory cytokines (such as IL-1 $\beta$, IL-6, and TNF- $\alpha$ ), that is, inhibiting the inflammatory stage of pulmonary fibrosis; it suppresses the expression of TGF- $\beta 1 /$ SNAI1 and SNAI2 to inhibit the development of EMT and regulates the proportion of MMPs/TIMPs to reduce the situation of pulmonary fibrosis itself.

3.4. Pulmonary Hypertension. Pulmonary hypertension $(\mathrm{PAH})$ is a chronic progressive disease with abnormally elevated pulmonary artery pressure that is characterized by increased pulmonary vascular resistance, leading to right heart failure and eventually death [74]. The key pathological change in $\mathrm{PAH}$ is the remodeling of small pulmonary arteries. This remodeling is characterized by thickening of the 
intima, media, and adventitia of small arteries, leading to the gradual narrowing of small pulmonary vessels, gradual increase in vascular resistance, and induction of adaptive right ventricular hypertrophy. The strain of constant pressure overload in the small arteries will eventually result in right heart failure [75]. Vascular remodeling in PAH is a complex and multifactorial process that involves not only perivascular inflammation, EMT, and pulmonary fibrosis but also impairs vasodilatory function mediated by pulmonary vascular endothelium [13, 76, 77].

Several studies have observed that FXR exists not only in pulmonary vascular endothelial cells but also in epithelial cells $[69,78]$. FXR plays an important role in maintaining lung function by inhibiting lung inflammatory response, producing lung surfactants, and promoting alveolar repair to resist lung injury. With regard to PAH $[48,79]$, FXR can inhibit the expression of the proinflammatory factor IL-6 and monocyte chemotactic protein-1 (MCP-1), as well as inhibit the NF- $\kappa \mathrm{B}$-mediated inflammatory response during $\mathrm{PAH}$ progression. Furthermore, FXR can inhibit the expression of TGF- $\beta 1$ to inhibit EMT and pulmonary fibrosis, thereby delaying the development of PAH. In addition, the bone morphogenetic protein/bone morphogenetic protein receptor (BMP/BMPR) system plays a vital role in PAH. The lack of BMPR expression in pulmonary vascular endothelial cells can result in the increased secretion of intracellular growth factors and IL-6, thereby promoting the occurrence of TGF- $\beta 1$-induced EMT, thus providing a circular response that aggravates the perivascular inflammation, EMT, and pulmonary vascular remodeling. FXR promotes the expression of BMP2/BMPR1A in pulmonary vascular endothelial cells, thereby improving the progress of PAH. Finally, FXR can regulate the balance of relaxation and contraction in smooth muscle cells by reducing the conduction of the NO-sGC-cGMP signaling pathway, which in turn normalizes the function of pulmonary vascular endothelium and thus inhibits the progression of PAH.

3.5. Acute Lung Injury and Acute Respiratory Distress Syndrome. Acute lung injury (ALI) and its more serious form, namely, acute respiratory distress syndrome (ARDS), are common diseases worldwide that may lead to acute respiratory failure and death [80]. The essence of ALI/ARDS is a variety of inflammatory cells, and their release of inflammatory factors and cytokines indirectly mediates lung inflammatory response, which can be considered as the pulmonary manifestation of systemic inflammatory response syndrome [81].

Several studies have confirmed that FXR mainly plays an important role in inhibiting lung inflammation and promoting lung regeneration in ALI/ARDS. On the one hand, FXR inhibits the release of proinflammatory cytokines (IL-1 $\beta$ and TNF- $\alpha$ ) and chemokines (CXCL1 and MCP-1) and partially upregulates the expression of anti-inflammatory cytokines (IL10 ) in the lungs to reduce the inflammatory response of ALI/ ARDS. ALI/ARDS induces the release of proinflammatory cytokines and chemokines by activating the NF- $\kappa \mathrm{B}$ signaling pathway, whereas FXR inhibits the NF- $\kappa$ B signaling pathway and also hinders MAPK and PI3K/Akt signaling pathways. In turn, FXR partially reduces the pulmonary inflammatory response of ALI/ARDS [82]. Notwithstanding, FXR activation inhibits the expression of P-selectin and induces the expression of the gene Foxm $1 b$, thereby improving the permeability of the lungs. Moreover, FXR activation inhibits the "chemotactic movement" of leukocytes from the peripheral circulation into the inflammatory tissues of the lungs. Furthermore, FXR activation stimulates the proliferation of endothelial cells to help restore the integrity of the pulmonary vascular endothelial barrier, thereby promoting the lung repair to inhibit ALI/ ARDS disease progression [47].

3.6. Non-Small-Cell Lung Cancer. Lung cancer is the leading cause of cancer-related deaths worldwide with the highest mortality rate among all malignant tumors [83]. Non-smallcell lung cancer (NSCLC) accounts for about $85 \%$ of all lung cancer cases [84]. A large number of studies have observed that numerous molecular changes and specific gene expressions are related to the occurrence and development of NSCLC $[85,86]$. Tumor cells can reshape the tumor microenvironment that will further affect the behavior and state of tumor cells $[87,88]$. The human lungs are susceptible to various poisons and pathogens. Consequently, the human lungs are prone to chronic damage and inflammation that form an inflammatory tumor microenvironment, thereby resulting in the occurrence of NSCLC [89].

FXR is regarded as a regulator of inflammation and immune response in immune-mediated disease subpopulations $[47,90]$. As a ligand-activated transcription factor, FXR can control the transcription of target genes by binding to FXR response elements (FXRE) [91]. Several studies have reported that, compared with that of healthy controls, the FXR of patients with NSCLC is markedly increased and its expression level is positively correlated with poor clinical results [92]. FXR can be recruited to the promoter of CCND1 in NSCLC cells and activate its transcription. CCND1 transcription can shorten the G1/S phase transition process in the cell cycle by upregulating the transcriptional expression of cyclin D1 and then promote the growth of NSCLC cells. FXR may also promote the development of NSCLC by regulating the tumor microenvironment, especially its immunological characteristics. In the NSCLC cell model, You et al. [93] found that knocking down FXR increases the expression of PD-L1, whereas the overexpression of FXR induces the downregulation of PD-L1 expression in NSCLC cells. These results indicated that FXR inhibits the expression of PD-L1 in NSCLC cells by binding to the putative FXRE in the PD-L1 promoter. This promoter could be used as a potential target for immunotherapy of $\mathrm{FXR}^{\text {high }} \mathrm{PD}-\mathrm{L} 1^{\text {low }}$ NSCLC. In summary, FXR plays a carcinogenic role in the progression of NSCLC.

\section{Conclusion}

As a multifunctional nuclear receptor, FXR can participate in the immune regulation and inflammation in the body, so it is highly expressed in the respiratory system with 
immune defense and prone to inflammation, such as bronchial epithelial cells, alveolar epithelial cells, alveolar macrophages, and pulmonary vascular endothelial cells. As a bile acid-activated nuclear receptor, FXR can bind to the FXRE and mediate anti-inflammatory and antifibrosis responses in the respiratory system by inhibiting the expression of proinflammatory cytokines and chemokines, texpression of COX-2 in the airways, toccurrence of EMT, and conduction of NF- $\kappa \mathrm{B}, \mathrm{MAPKs}$, and PI3K/Akt signaling pathways. In addition, FXR can regulate the tumor microenvironment by regulating the balance of inflammatory and immune responses in the body to promote the occurrence and development of NSCLC. Thus, it can be a potential target for immunotherapy of NSCLC.

In summary, FXR is expressed in the respiratory system and plays an important pathophysiological function in respiratory diseases. FXR regulates the bile acid metabolism in the body and also exerts its anti-inflammatory and antifibrotic effects in the airways and the lungs. Further research on the role of FXR in the pathogenesis of various respiratory diseases would aid in developing this receptor as an indicator of the progression of these diseases and as their potential therapeutic target. It is believed that FXR can play a pivotal role in respiratory diseases in the near future.

\section{Abbreviations}

$\begin{array}{ll}\text { FXR: } & \text { Farnesoid X receptor } \\ \text { LBD: } & \text { Ligand-binding domain } \\ \text { DBD: } & \text { DNA-binding domain } \\ \text { LPS: } & \text { Lipopolysaccharide } \\ \text { COPD: } & \text { Chronic obstructive pulmonary disease } \\ \text { EMT: } & \text { Epithelial-mesenchymal transition } \\ \text { COX-2: } & \text { Cyclooxygenase } 2 \\ \text { IL-4: } & \text { Interleukin-4 } \\ \text { IL-5: } & \text { Interleukin-5 } \\ \text { IL-13: } & \text { Interleukin-13 } \\ \text { NF- } \kappa \text { B: } & \text { Nuclear factor-kappaB } \\ \text { TNF- } \alpha: & \text { Tumor necrosis factor- } \alpha \\ \text { IPF: } & \text { Idiopathic pulmonary fibrosis } \\ \text { TGF- } \beta 1: & \text { Transforming growth factor- } \beta 1 \\ \text { SNAI1: } & \text { Zinc finger transcription factors } 1 \\ \text { SNAI2: } & \text { Zinc finger transcription factors } 2 \\ \text { AECIs: } & \text { Alveolar epithelial type I cells } \\ \text { ATECIIs: } & \text { Alveolar epithelial type II cells } \\ \text { MMP: } & \text { Matrix metalloproteinases } \\ \text { TIMP: } & \text { Tissue inhibitor of metalloproteinases } \\ \text { PAH: } & \text { Pulmonary hypertension } \\ \text { MCP-1: } & \text { Monocyte chemotactic protein-1 } \\ \text { BMP: } & \text { Bone morphogenetic protein } \\ \text { BMPR: } & \text { Bone morphogenetic protein receptor } \\ \text { ALI: } & \text { Acute lung injury } \\ \text { ARDS: } & \text { Acute respiratory distress syndrome } \\ \text { NSCLC: } & \text { Non-small-cell lung cancer } \\ \text { FXRE: } & \text { FXR response elements. } \\ & \end{array}$

\section{Data Availability}

No data were used to support this study.

\section{Conflicts of Interest}

The authors declare no conflicts of interest regarding the article.

\section{Authors' Contributions}

Jin-nan $\mathrm{Wu}$ was responsible for the conceptualization, software, investigation, formal analysis, data curation, and writing and preparation of the original draft; Jin-liang Chen was responsible for the methodology, visualization, investigation, supervision, software, and validation; Jian-rong Chen was responsible for project administration and writing, reviewing, and editing the manuscript.

\section{Acknowledgments}

This work was supported by the following grants: Postgraduate Research and Practice Innovation Program of Jiangsu Province (grant no. SJCX19_0873); the Natural Science Foundation of Jiangsu Province (Grant no. BK20191207); the Science and Technology Program of Nantong City (grant no. HS2018002). The authors thank all the staff members in the institution.

\section{References}

[1] S. Modica, R. M. Gadaleta, and A. Moschetta, "Deciphering the nuclear bile acid receptor FXR paradigm," Nuclear Receptor Signaling, vol. 8, 2010.

[2] D.-J. Shin and L. Wang, "Bile acid-activated receptors: a review on FXR and other nuclear receptors," Bile Acids and Their Receptors, vol. 256, pp. 51-72, 2019.

[3] G. Musso, R. Gambino, and M. Cassader, "Cholesterol metabolism and the pathogenesis of non-alcoholic steatohepatitis," Progress in Lipid Research, vol. 52, no. 1, pp. 175-191, 2013.

[4] R. Sun, N. Yang, B. Kong et al., "Orally administered berberine modulates hepatic lipid metabolism by altering microbial bile acid metabolism and the intestinal FXR signaling pathway," Molecular Pharmacology, vol. 91, no. 2, pp. 110-122, 2017.

[5] Y. Hou, W. Fan, W. Yang, A. Q. Samdani, A. O. Jackson, and S. Qu, "Farnesoid X receptor: an important factor in blood glucose regulation," Clinica Chimica Acta, vol. 495, pp. 29-34, 2019.

[6] A. Di Ciaula, D. Q.-H. Wang, E. Molina-Molina et al., "Bile acids and cancer: direct and environmental-dependent effects," Annals of Hepatology, vol. 16, pp. s87-s105, 2017.

[7] V. Keitel, C. Dröge, and D. Häussinger, "Targeting FXR in cholestasis," Bile Acids and Their Receptors, vol. 256, pp. 299-324, 2019.

[8] G. Alpini, R. Tariq, and A. K. Singal, "FXR deficiency and alcoholic liver disease: tissue is the issue," Digestive and Liver Disease, vol. 51, no. 4, pp. 577-578, 2019.

[9] P. J. Trivedi, G. M. Hirschfield, and M. E. Gershwin, "Obeticholic acid for the treatment of primary biliary cirrhosis," Expert Review of Clinical Pharmacology, vol. 9, no. 1, pp. 13-26, 2016.

[10] L. Ning, X. Lou, F. Zhang, and G. Xu, "Nuclear receptors in the pathogenesis and management of inflammatory bowel disease," Mediators Inflamm, vol. 2019, Article ID 2624941, 13 pages, 2019. 
[11] D. Moris, C. Giaginis, G. Tsourouflis et al., "Farnesoid-X receptor (FXR) as a promising pharmaceutical target in atherosclerosis," Current Medicinal Chemistry, vol. 24, no. 11, pp. 1147-1157, 2017.

[12] T. Fu, S. Coulter, E. Yoshihara et al., "FXR regulates intestinal cancer stem cell proliferation," Cell, vol. 176, no. 5, pp. 1098-1112, 2019.

[13] P. Comeglio, A. Morelli, L. Adorini, M. Maggi, and L. Vignozzi, "Beneficial effects of bile acid receptor agonists in pulmonary disease models," Expert Opinion on Investigational Drugs, vol. 26, no. 11, pp. 1215-1228, 2017.

[14] F. J. Gonzalez, C. Jiang, and A. D. Patterson, "An intestinal microbiota-farnesoid X receptor Axis modulates metabolic disease," Gastroenterology, vol. 151, no. 5, pp. 845-859, 2016.

[15] M. Bhargava, K. J. Viken, S. Dey et al., "Proteome profiling in lung injury after hematopoietic stem cell transplantation," Biology of Blood and Marrow Transplantation, vol. 22, no. 8, pp. 1383-1390, 2016.

[16] E. L. Yang, B. Q. Hu, W. Wu et al., "Alveolar type II epithelial cell dysfunction in rat experimental hepatopulmonary syndrome (HPS)," PLoS One, vol. 9, no. 11, 2014.

[17] D. You, L. E. Lyn-Cook, D. M. Gatti et al., "Nitrosative stress and lipid homeostasis as a mechanism for zileuton hepatotoxicity and resistance in genetically sensitive mice," Toxicological Sciences, vol. 175, no. 2, pp. 220-235, 2020.

[18] B. M. Forman, E. Goode, J. Chen et al., "Identification of a nuclear receptor that is activated by farnesol metabolites," Cell, vol. 81, no. 5, pp. 687-693, 1995.

[19] H. Wang, Q. He, G. Wang, X. Xu, and H. Hao, "FXR modulators for enterohepatic and metabolic diseases," Expert Opinion on Therapeutic Patents, vol. 28, no. 11, pp. 765-782, 2018.

[20] S. Fiorucci and E. Distrutti, "Bile acid-activated receptors, intestinal microbiota, and the treatment of metabolic disorders," Trends in Molecular Medicine, vol. 21, no. 11, pp. 702-714, 2015.

[21] Y. Zhang, H. R. Kast-Woelbern, and P. A. Edwards, "Natural structural variants of the nuclear receptor farnesoid $\mathrm{X}$ receptor affect transcriptional activation," Journal of Biological Chemistry, vol. 278, no. 1, pp. 104-110, 2003.

[22] J. S. Teodoro, A. P. Rolo, and C. M. Palmeira, "Hepatic FXR: key regulator of whole-body energy metabolism," Trends in Endocrinology \& Metabolism, vol. 22, no. 11, pp. 458-466, 2011.

[23] Y.-D. Wang, W.-D. Chen, D. D. Moore, and W. Huang, "FXR: a metabolic regulator and cell protector," Cell Research, vol. 18, no. 11, pp. 1087-1095, 2008.

[24] G. A. Preidis, K. H. Kim, and D. D. Moore, "Nutrient-sensing nuclear receptors PPAR $\alpha$ and FXR control liver energy balance," Journal of Clinical Investigation, vol. 127, no. 4, pp. 1193-1201, 2017.

[25] E. S. Friedman, Y. Li, T.-C. D. Shen et al., "FXR-dependent modulation of the human small intestinal microbiome by the bile acid derivative obeticholic acid," Gastroenterology, vol. 155, no. 6, pp. 1741-1752, 2018.

[26] M. Herman-Edelstein, T. Weinstein, and M. Levi, "Bile acid receptors and the kidney," Current Opinion in Nephrology and Hypertension, vol. 27, no. 1, pp. 56-62, 2018.

[27] H. Lee, Y. Zhang, F. Y. Lee, S. F. Nelson, F. J. Gonzalez, and P. A. Edwards, "FXR regulates organic solute transporters $\alpha$ and $\beta$ in the adrenal gland, kidney, and intestine," Journal of Lipid Research, vol. 47, no. 1, pp. 201-214, 2006.

[28] R. Zhang, L. Peng, H. Ran et al., "Farnesoid X receptor activation modulates calcium homeostasis in rat aortic vascular smooth muscle cells," The Chinese Journal of Physiology, vol. 61, no. 4, pp. 210-220, 2018.

[29] M. A. Olsson, A. T. García-Sosa, and U. Ryde, "Binding affinities of the farnesoid X receptor in the D3R Grand Challenge 2 estimated by free-energy perturbation and docking," Journal of Computer-Aided Molecular Design, vol. 32, no. 1, pp. 211-224, 2018.

[30] V. Hiebl, A. Ladurner, S. Latkolik, and V. M. Dirsch, "Natural products as modulators of the nuclear receptors and metabolic sensors LXR, FXR and RXR," Biotechnology Advances, vol. 36, no. 6, pp. 1657-1698, 2018.

[31] W. Zheng, Y. Lu, S. Tian et al., "Structural insights into the heterodimeric complex of the nuclear receptors FXR and RXR," Journal of Biological Chemistry, vol. 293, no. 32, pp. 12535-12541, 2018.

[32] J. Liu, H. Lu, Y.-F. Lu et al., "Potency of individual bile acids to regulate bile acid synthesis and transport genes in primary human hepatocyte cultures," Toxicological Sciences, vol. 141, no. 2, pp. 538-546, 2014.

[33] K. F. Chambers, P. E. Day, H. T. Aboufarrag, and P. A. Kroon, "Polyphenol effects on cholesterol metabolism via bile acid biosynthesis, CYP7A1: a review," Nutrients, vol. 11, no. 11, p. 2588, 2019.

[34] D. Rizzolo, K. Buckley, B. Kong et al., "Bile acid homeostasis in a cholesterol $7 \alpha$-hydroxylase and sterol 27-hydroxylase double knockout mouse model," Hepatology (Baltimore, MD), vol. 70, no. 1, pp. 389-402, 2019.

[35] M. C. Cave, H. B. Clair, J. E. Hardesty et al., "Nuclear receptors and nonalcoholic fatty liver disease," Biochimica et Biophysica Acta (BBA)-Gene Regulatory Mechanisms, vol. 1859, no. 9, pp. 1083-1099, 2016.

[36] K. E. Zaborska and B. P. Cummings, "Rethinking bile acid metabolism and signaling for type 2 diabetes treatment," Current Diabetes Reports, vol. 18, no. 11, p. 109, 2018.

[37] Y. Zhang, F. Y. Lee, G. Barrera et al., "Activation of the nuclear receptor FXR improves hyperglycemia and hyperlipidemia in diabetic mice," Proceedings of the National Academy of Sciences, vol. 103, no. 4, pp. 1006-1011, 2006.

[38] M. Camilleri, "Bile Acid diarrhea: prevalence, pathogenesis, and therapy," Gut Liver, vol. 9, no. 3, pp. 332-339, 2015.

[39] G. Alvarez-Sola, I. Uriarte, M. U. Latasa et al., "Bile acids, FGF15/19 and liver regeneration: from mechanisms to clinical applications," Biochimica et Biophysica Acta (BBA)-Molecular Basis of Disease, vol. 1864, no. 4, pp. 1326-1334, 2018.

[40] U. Deuschle, J. Schüler, A. Schulz et al., "FXR controls the tumor suppressor NDRG2 and FXR agonists reduce liver tumor growth and metastasis in an orthotopic mouse xenograft model," PLoS One, vol. 7, no. 10, 2012.

[41] X. X. Wang, Y. Luo, D. Wang et al., "A dual agonist of farnesoid X receptor (FXR) and the G protein-coupled receptor TGR5, INT-767, reverses age-related kidney disease in mice," Journal of Biological Chemistry, vol. 292, no. 29, pp. 12018-12024, 2017.

[42] X. X. Wang, D. Wang, Y. Luo et al., "FXR/TGR5 dual agonist prevents progression of nephropathy in diabetes and obesity," Journal of the American Society of Nephrology, vol. 29, no. 1, pp. 118-137, 2018.

[43] X. Chen, F. Lu, G. Luo et al., "Discovery of selective farnesoid $\mathrm{X}$ receptor agonists for the treatment of hyperlipidemia from traditional Chinese medicine based on virtual screening and in vitro validation," Journal of Biomolecular Structure and Dynamics, vol. 38, no. 15, pp. 4461-4470, 2019.

[44] D. Bishop-Bailey, D. T. Walsh, and T. D. Warner, "Expression and activation of the farnesoid X receptor in the vasculature," 
Proceedings of the National Academy of Sciences, vol. 101, no. 10 , pp. 3668-3673, 2004.

[45] Y. T. Y. Li, K. E. Swales, G. J. Thomas, T. D. Warner, and D. Bishop-Bailey, "Farnesoid $\mathrm{X}$ receptor ligands inhibit vascular smooth muscle cell inflammation and migration," Arteriosclerosis, Thrombosis, and Vascular Biology, vol. 27, no. 12, pp. 2606-2611, 2007.

[46] S. M. Hendrick, M. S. Mroz, C. M. Greene, S. J. Keely, and B. J. Harvey, "Bile acids stimulate chloride secretion through CFTR and calcium-activated Cl- channels in Calu-3 airway epithelial cells," American Journal of Physiology-Lung Cellular and Molecular Physiology, vol. 307, no. 5, pp. L407-L418, 2014.

[47] L. Zhang, T. Li, D. Yu, B. M. Forman, and W. Huang, "FXR protects lung from lipopolysaccharide-induced acute injury," Molecular Endocrinology, vol. 26, no. 1, pp. 27-36, 2012.

[48] P. Comeglio, S. Filippi, E. Sarchielli et al., "Therapeutic effects of the selective farnesoid $\mathrm{X}$ receptor agonist obeticholic acid in a monocrotaline-induced pulmonary hypertension rat model," Journal of Endocrinological Investigation, vol. 42, no. 8, pp. 951-965, 2019.

[49] J. C. Hogg, F. Chu, S. Utokaparch et al., "The nature of smallairway obstruction in chronic obstructive pulmonary disease," New England Journal of Medicine, vol. 350, no. 26, pp. 2645-2653, 2004.

[50] S. Sohal and E. Walters, "Role of epithelial mesenchymal transition (EMT) in chronic obstructive pulmonary disease (COPD)," Respiratory Research, vol. 14, no. 1, p. 120, 2013.

[51] S. T. Gohy, C. Hupin, C. Fregimilicka et al., "Imprinting of the COPD airway epithelium for dedifferentiation and mesenchymal transition," European Respiratory Journal, vol. 45, no. 5, pp. 1258-1272, 2015.

[52] A. Xaubet, J. Roca-Ferrer, L. Pujols et al., "Cyclooxygenase-2 is up-regulated in lung parenchyma of chronic obstructive pulmonary disease and down-regulated in idiopathic pulmonary fibrosis," Sarcoidosis Vasculitis and Diffuse Lung Diseases, vol. 21, no. 1, pp. 35-42, 2004.

[53] Y. Chen, P. Chen, M. Hanaoka et al., "Enhanced levels of prostaglandin E2 and matrix metalloproteinase-2 correlate with the severity of airflow limitation in stable COPD," Respirology, vol. 13, no. 7, pp. 1014-1021, 2008.

[54] B. Chen, W. J. You, S. Xue et al., "Overexpression of farnesoid $\mathrm{X}$ receptor in small airways contributes to epithelial to mesenchymal transition and COX-2 expression in chronic obstructive pulmonary disease," Journal of Thoracic Disease, vol. 8, no. 11, pp. 3063-3074, 2016.

[55] J. W. Mims, "Asthma: definitions and pathophysiology," International Forum of Allergy \& Rhinology, vol. 5, no. 1, pp. s2-s6, 2015.

[56] A. kleinJan, "Airway inflammation in asthma: key players beyond the Th2 pathway," Current Opinion in Pulmonary Medicine, vol. 22, no. 1, pp. 46-52, 2016.

[57] P. Bradding and G. Arthur, "Mast cells in asthma-state of the art," Clinical \& Experimental Allergy, vol. 46, no. 2, pp. 194-263, 2016.

[58] C. Draijer and M. Peters-Golden, "Alveolar macrophages in allergic asthma: the forgotten cell awakes," Current Allergy and Asthma Reports, vol. 17, no. 2, p. 12, 2017.

[59] A. Imanifooladi, S. Yazdani, and M. Nourani, "The role of nuclear factor- $\kappa \mathrm{b}$ in inflammatory lung disease," Inflammation \& Allergy-Drug Targets, vol. 9, no. 3, pp. 197-205, 2010.

[60] Y. M. W. Janssen-Heininger, M. E. Poynter, S. W. Aesif et al., "Nuclear factor B, airway epithelium, and asthma: avenues for redox control," Proceedings of the American Thoracic Society, vol. 6, no. 3, pp. 249-255, 2009.

[61] F. B. Shaik, K. Panati, V. R. Narasimha, and V. R. Narala, "Chenodeoxycholic acid attenuates ovalbumin-induced airway inflammation in murine model of asthma by inhibiting the T H 2 cytokines," Biochemical and Biophysical Research Communications, vol. 463, no. 4, pp. 600-605, 2015.

[62] G. Sgalla, A. Biffi, and L. Richeldi, "Idiopathic pulmonary fibrosis: diagnosis, epidemiology and natural history," Respirology, vol. 21, no. 3, pp. 427-437, 2016.

[63] B. Crestani, S. Marchand-Adam, A. Fabre et al., "Mechanisms in pulmonary fibrosis," Rev Prat, vol. 57, no. 20, pp. 22222226, 2007.

[64] B. Crestani, "Pathophysiology of idiopathic pulmonary fibrosis," Bulletin de l'Académie Nationale de Médecine, vol. 194, no. 2, pp. 343-351, 2010.

[65] L. Zhu, X. Fu, X. Chen, X. Han, and P. Dong, "M2 macrophages induce EMT through the TGF- $\beta /$ Smad2 signaling pathway," Cell Biology International, vol. 41, no. 9, pp. 960-968, 2017.

[66] M. K. Jolly, C. Ward, M. S. Eapen et al., "Epithelial-mesenchymal transition, a spectrum of states: role in lung development, homeostasis, and disease," Developmental Dynamics, vol. 247 , no. 3, pp. 346-358, 2018.

[67] K. M. Hajra, D. Y. Chen, and E. R. Fearon, "The SLUG zincfinger protein represses E-cadherin in breast cancer," Cancer Research, vol. 62, no. 6, pp. 1613-1618, 2002.

[68] V. Bolós, H. Peinado, M. A. Pérez-Moreno et al., "The transcription factor Slug represses E-cadherin expression and induces epithelial to mesenchymal transitions: a comparison with Snail and E47 repressors," Journal of Cell Science, vol. 116, no. 3, pp. 499-511, 2003.

[69] J. Wang, Y. Huang, X. Hou et al., "Morphologic damage of rat alveolar epithelial type II cells induced by bile acids could Be ameliorated by farnesoid X receptor inhibitor Z-guggulsterone in vitro," Biomed Res Int, vol. 2016, Article ID 9283204, 9 pages, 2016.

[70] C. Hill, M. Jones, D. Davies, and Y. Wang, "Epithelial-mesenchymal transition contributes to pulmonary fibrosis via aberrant epithelial/fibroblastic cross-talk," Journal of Lung Health and Diseases, vol. 3, no. 2, pp. 31-35, 2019.

[71] C.-H. Yen, S. Leu, Y.-C. Lin et al., "Sildenafil limits monocrotaline-induced pulmonary hypertension in rats through suppression of pulmonary vascular remodeling," Journal of Cardiovascular Pharmacology, vol. 55, no. 6, pp. 574-584, 2010.

[72] P. Comeglio, S. Filippi, E. Sarchielli et al., "Anti-fibrotic effects of chronic treatment with the selective FXR agonist obeticholic acid in the bleomycin-induced rat model of pulmonary fibrosis," The Journal of Steroid Biochemistry and Molecular Biology, vol. 168, pp. 26-37, 2017.

[73] C. A. Fielding, G. W. Jones, R. M. McLoughlin et al., "Interleukin-6 signaling drives fibrosis in unresolved inflammation," Immunity, vol. 40, no. 1, pp. 40-50, 2014.

[74] M. Rabinovitch, C. Guignabert, M. Humbert, and M. R. Nicolls, "Inflammation and immunity in the pathogenesis of pulmonary arterial hypertension," Circulation Research, vol. 115, no. 1, pp. 165-175, 2014.

[75] T. Thenappan, M. L. Ormiston, J. J. Ryan, and S. L. Archer, "Pulmonary arterial hypertension: pathogenesis and clinical management," BMJ, vol. 360, p. j5492, 2018.

[76] J. Leopold and B. Maron, "Molecular mechanisms of pulmonary vascular remodeling in pulmonary arterial hypertension," International Journal of Molecular Sciences, vol. 17, no. 5, p. 761, 2016. 
[77] M. Guazzi, S. A. Phillips, R. Arena, and C. J. Lavie, "Endothelial dysfunction and lung capillary injury in cardiovascular diseases," Progress in Cardiovascular Diseases, vol. 57, no. 5, pp. 454-462, 2015.

[78] F. He, J. Li, Y. Mu et al., "Downregulation of endothelin-1 by farnesoid X receptor in vascular endothelial cells," Circulation Research, vol. 98, no. 2, pp. 192-199, 2006.

[79] L. Vignozzi, A. Morelli, I. Cellai et al., "Cardiopulmonary protective effects of the selective FXR agonist obeticholic acid in the rat model of monocrotaline-induced pulmonary hypertension," The Journal of Steroid Biochemistry and Molecular Biology, vol. 165, pp. 277-292, 2017.

[80] E. R. Johnson and M. A. Matthay, "Acute lung injury: epidemiology, pathogenesis, and treatment," Journal of Aerosol Medicine and Pulmonary Drug Delivery, vol. 23, no. 4, pp. 243-252, 2010.

[81] Y. Butt, A. Kurdowska, and T. C. Allen, "Acute lung injury: a clinical and molecular review," Archives of Pathology \& Laboratory Medicine, vol. 140, no. 4, pp. 345-350, 2016.

[82] J. Fei, L. Fu, B. Hu et al., "Obeticholic acid alleviate lipopolysaccharide-induced acute lung injury via its anti-inflammatory effects in mice," International Immunopharmacology, vol. 66, pp. 177-184, 2019.

[83] R. L. Siegel, K. D. Miller, and A. Jemal, "Cancer statistics, 2020," CA: A Cancer Journal for Clinicians, vol. 70, no. 1, pp. 7-30, 2020.

[84] M. Zheng, "Classification and pathology of lung cancer," Surgical Oncology Clinics of North America, vol. 25, no. 3, pp. 447-468, 2016.

[85] M. G. Kris, B. E. Johnson, L. D. Berry et al., "Using multiplexed assays of oncogenic drivers in lung cancers to select targeted drugs," JAMA, vol. 311, no. 19, pp. 1998-2006, 2014.

[86] Q. Liu, Z. Yu, Y. Xiang et al., "Prognostic and predictive significance of thymidylate synthase protein expression in non-small cell lung cancer: a systematic review and metaanalysis," Cancer Biomarkers, vol. 15, no. 1, pp. 65-78, 2015.

[87] D. Spano and M. Zollo, "Tumor microenvironment: a main actor in the metastasis process," Clinical \& Experimental Metastasis, vol. 29, no. 4, pp. 381-395, 2012.

[88] Y. Wu, S. Antony, J. L. Meitzler, and J. H. Doroshow, "Molecular mechanisms underlying chronic inflammationassociated cancers," Cancer Letters, vol. 345, no. 2, pp. 164-173, 2014.

[89] M. Zhao, Y. Liu, R. Liu et al., "Upregulation of IL-11, an IL-6 family cytokine, promotes tumor progression and correlates with poor prognosis in non-small cell lung cancer," Cellular Physiology and Biochemistry, vol. 45, no. 6, pp. 2213-2224, 2018.

[90] P. Vavassori, A. Mencarelli, B. Renga, E. Distrutti, and S. Fiorucci, "The bile acid receptor FXR is a modulator of intestinal innate immunity," The Journal of Immunology, vol. 183, no. 10, pp. 6251-6261, 2009.

[91] B. Renga, C. D’Amore, S. Cipriani et al., "FXR mediates a chromatin looping in the GR promoter thus promoting the resolution of colitis in rodents," Pharmacological Research, vol. 77, pp. 1-10, 2013.

[92] W. You, B. Chen, X. Liu et al., "Farnesoid X receptor, a novel proto-oncogene in non-small cell lung cancer, promotes tumor growth via directly transactivating CCND1," Scientific Reports, vol. 7, p. 591, 2017.

[93] W. You, L. Li, D. Sun et al., "Farnesoid X receptor constructs an immunosuppressive microenvironment and sensitizes FXRhighPD-11low NSCLC to anti-PD-1 immunotherapy," Cancer Immunology Research, vol. 7, no. 6, pp. 990-1000, 2019. 